\title{
SPACE TIME COVARIANCE OF CANONICAL QUANTIZATION OF GRAVITY: A (FORMAL) GENERAL RESULT AND THE (RIGOROUS) EXPLICIT CASE OF 2+1 QUANTUM COSMOLOGY
}

\author{
Christodoulakis, T. and G.O. Papadopoulosy \\ Department of Physics, University of Athens, \\ Nuclear and Particle Physics Section, Panepistimioupolis, Ilisia GR 157-71, Athens, Hellas, Greece
}

Received 2013-10-19, Revised 2013-12-09; Accepted 2014-01-27

\begin{abstract}
A general classical theorem is presented according to which all invariant relations among the space time metric scalars, when turned into functions on the Phase Space of full Pure Gravity (using the Canonical Equations of motion), be-come weakly vanishing functions of the Quadratic and Linear Constraints. The implication of this result is that (formal) Dirac consistency of the Quantum Operator Constraints (annihilating the wave Function) suffices to guarantee space time covariance of the ensuing quantum theory: An ordering for each invariant relation will always exist such that the emanating operator has an eigenvalue identical to the classical value. The example of $2+1$ Quantum Cosmology is explicitly considered: The four possible "Cosmological Solutions" -two for pure Einstein's equations plus two more when a $\Lambda$ term is present- are exhibited and the corresponding models are quantized. The invariant relations describing the geometries are explicitly calculated and promoted to operators whose eigenvalues are their corresponding classical values.
\end{abstract}

Keywords: Space Time, Gravity, Quantum Cosmology, Canonical Quantization

\section{INTRODUCTION}

The problem of space time covariance of a Quantum Theory of Gravity (Isham, 1995) within the context of Canonical Quantization can essentially be described as follows: Classically, Einstein's Field equations are known to be (not manifestly but explicitly) equivalent to the Hamiltonian and Momentum constraints plus the Canonical Equations of motion. This is understandable since, although the canonical analysis uses objects defined on the hypersurface, the momenta involve the extrinsic curvature and thus carry the information of the embedding of the hypersurface in space time. When we canonically quantize, the momenta become functional derivatives with respect to the spatial metric (choice of Polarization in the phase space) and thus the fate of space time covariance is somewhat obscure. Of course if one takes special care so that the geometrical meaning of the classical constraints is maintained at the quantum level, one is justified to expect space time covariance of the ensuing theory. Indeed as we shall see in the first section, when we suitably define the equivalence between two sets of state vectors emerging upon quantizing the same space time in two different foliations, then space time covariance is indeed achieved.

In the next sections we present the example of $2+1$ spatially homogeneous cosmological models in the absence as well as in the presence of a $\Lambda$ term. The classical solutions i.e., the different "cosmological" disguises of Minkowski space time or the maximally symmetric space are explicitly given. The corresponding models are quantized and the classical observables are

Corresponding Author: Christodoulakis, T., Department of Physics, University of Athens, Nuclear and Particle Physics Section, Panepistimioupolis, Ilisia GR 157-71, Athens, Hellas, Greece 
turned into operators in such a way that their eigenvalues coincide with the classical values. In this sense it is proven that the two pairs of state vectors are equivalent.

\section{A CLASSICAL THEOREM AND ITS QUANTUM IMPLICATIONS}

As is well known (Sundermeyer, 1982), the canonical analysis of pure Gravity consists in the following statements Equation (2.1a to e):

$$
\begin{aligned}
& \mathrm{H}_{0}\left(\mathrm{~g}_{\mathrm{ij}}, \pi^{\mathrm{ij}}\right) \approx 0 \\
& \mathrm{H}_{\mathrm{k}}\left(\mathrm{g}_{\mathrm{ij}}, \pi^{\mathrm{ij}}\right) \approx 0 \\
& \dot{\mathrm{g}}_{\mathrm{ij}}=\left\{\mathrm{g}_{\mathrm{ij}}, \mathrm{H}\right\} \\
& \dot{\pi}_{\mathrm{ij}}=\left\{\pi^{\mathrm{ij}}, \mathrm{H}\right\} \\
& \mathrm{H}=\int\left(\mathrm{N}^{0} \mathrm{H}_{0}+\mathrm{N}^{\mathrm{k}} \mathrm{H}_{\mathrm{k}}\right) \mathrm{d}^{3} \mathrm{x}
\end{aligned}
$$

Which are explicitly equivalent to the ten Einstein's Field Equations. If we adopt the notion that classical observables, are all the geometrical objects that do not depend on the gauge, i.e. the coordinate system, then we are led to identify these observables with invariant relations among space time scalars. These scalars can be constructed in two ways: Firstly, by contracting all the indices of tensor products of the Riemann tensor and its covariant derivatives of any order. Secondly, in the case of spacetimes admitting a null, covariantly constant vector field (pp waves where all the scalars constructed in the above mentioned way are identically vanishing), by finding proportionality factors between tensors constructed by the Riemann tensor and its covariant derivatives of any order. Anyway, the scalars themselves do not describe the space time in an invariant manner since their functional form in terms of the coordinates changes when the coordinate system is altered. A way to generate invariant relations among these scalars, albeit not the most efficient one, would be to take a base of 4 scalars (say $\mathrm{Q}_{1}, \ldots \mathrm{Q}_{4}$ ) and solve for the coordinates. Then, any other scalar (say $\mathrm{Q}_{5}$ ) becomes expressible in terms of the 4 scalars chosen $\left(Q_{5}=f\left(Q_{1}, \ldots, Q_{4}\right)\right)$. Now this relation is characteristic of the geometry i.e., does not change form under coordinate transformations. In this sense a geometry is complectly characterized by a set of relations Equation (2.2):

$$
\mathrm{f}^{\mathrm{A}}\left(\mathrm{Q}_{\mathrm{i}}\right)=0
$$

The index A is at most countable. Turning these relations into functions on the phase space we notice that they become weakly vanishing quantities Equation (2.3):

$\mathrm{h}^{\mathrm{A}}\left(\mathrm{g}_{\mathrm{ij}}, \mathrm{p}^{\mathrm{ij}}\right) \gg 0$

In implementing this step use has been made of canonical equations of motion in order to substitute all higher time derivatives of the metric of the slice. But at this point, we invoke a known theorem of constrained dynamics (Sundermeyer, 1982):

\section{Theorem}

"Every weakly vanishing function in Phase Space is strongly equal to some expression containing the Constraints (which define a surface in Phase Space)"

Moreover the expression under discussion ought to vanish on-mass shell (i.e., when the constraints are set to zero). Thus Equation (2.4):

$\mathrm{h}^{\mathrm{A}}=\mathrm{h}^{\mathrm{A}}\left(\mathrm{H}_{0}, \mathrm{H}_{\mathrm{k}}\right)$

The translation of the above result in the velocity phase space reads as follows: Consider an invariant relation $\mathrm{f}^{\mathrm{A}}\left(\mathrm{Q}_{\mathrm{i}}\right)=0$ of any space time geometry which satisfies Einstein's Field Equations. Evaluate the lefthand side for the generic space time metric. Then, eliminate from the resulting expression all higher time derivatives of the metric (on the slice) using the spatial Einstein equations (and their time derivatives of the appropriate order). The end result will be that $\mathrm{f}^{\mathrm{A}}$ will become such a function of the $G_{0}^{0}, G_{k}^{0} k$ constraints so that it vanishes when the constraints are set to zero. The proof rests on the fact that the constraints are the only quantities of the spatial metric and its time derivative that vanish, a thing that is guaranteed by the consistency of the Field Equations; Any other function on the velocity Phase Space which vanishes by virtue of the Field Equations, such as $\mathrm{f}^{\mathrm{A}}$, is necessarily expressible in terms of the constraints.

The implications of this for the quantum theory are obvious and important: Adopting the point of view that the quantum observables are to be the operator analogues of the classical observables, we are assured that, for each and every such observable, there will exist a factor ordering such that all ensuing operators, when acting on the appropriate states defined by Equation (2.5a and $b$ ): 


$$
\hat{\mathrm{H}}_{0} \Psi=0
$$

$$
\hat{\mathrm{H}}_{\mathrm{k}} \Psi=0
$$

Will have vanishing eigenvalues Equation (2.6):

$$
\hat{\mathrm{h}}^{\mathrm{A}} \Psi=0
$$

This establishes the (formal) space time covariance of the quantum theory described by (2.5) above.

\section{PRELIMINARIES ON SPATIALLY HOMOGENEOUS SPACE-TIMES}

We are now interested in exemplifying the theorem and its implications by means of a concrete, finitedimensional Quantum Cosmology example. To this end, we wish to manufacture a situation in which a specific space time admits two different homogeneous foliations. This is generically impossible in $3+1$ Cosmology, where the imposition, on the hypersurface, of the various 3-dim Lie Groups as symmetry groups of motion, leads to the distinct Bianchi models. The very rare exceptions have to do with limiting cases, such as Milne's solution (Milne, 1932) which is 4-dim Minkowski geometry disguised as a Type V cosmology, or the trivial case of Kasner's universes with two of the three scale factors constant. A suitable situation that comes to mind is the case of 2+1 dimensions: There, the pure Einstein's Field Equations admit only the Minkowski geometry as a solution, while when a $\Lambda$ term is present, the only possibility is a maximally symmetric space. Thus, if we solve the general Field Equations under the restriction of spatial homogeneity, we are bound to end up with "cosmological" descriptions of either Minkowski geometry or a space of maximal symmetry, correspondingly.

Before proceeding with the examples, it is deemed as appropriate to exhibit some basic assumptions.

Spacetime, is assumed to be the pair $(\mathrm{M}, \mathrm{g})$ where $\mathrm{M}$ is a 3-dimensional, Hausdorff, connected, time-oriented and $\mathrm{C} 1$ manifold and $\mathrm{g}$ is a $(0,2)$ tensor field, globally defined, $\mathrm{C} 1$, non degenerate and Lorentzian i.e., it has signature $(-,+,+)$. In the spirit of $2+1$ analysis, $M=R \times \Sigma_{t}$, where the 2-dimensional orientable sub manifolds $\Sigma_{\mathrm{t}}$ (surfaces of simultaneity), are spacelike surfaces of constant time; their evolution in time, results in the entire spacetime. The assumption of spatial homogeneity corresponds to the imposition of the action of a symmetry group of transformations $G$ upon the manifolds $\Sigma_{\mathrm{t}}$. Usually the group $\mathrm{G}$ is not only continuous, but also a Lie group -thus denoted by $G_{r}$, where $r$ is the dimension of the space of its parameters. Avoiding the details on these issues \{these matters can be easily found in every standard reference see e.g., (Eisenhart, 1933)- we simply state that spatially homogeneous models with a simply transitive action of the symmetry group are described (apart the topology of $\Sigma_{\mathrm{t}}$ ) by an invariant basis of one forms $\sigma_{\mathrm{i}}^{\alpha}(\mathrm{x}) \mathrm{dx}{ }^{\mathrm{i}}$. By invariant we mean that their Lie Derivative with respect to the generators of the Lie Group $G_{r}$ are zero. The case of Groups which act multiply transitively will not concern us.

The line element of such a space time can be casted into the form Equation (3.1):

$$
\begin{aligned}
& d s^{2}=\left(N^{\alpha}(t) N_{\alpha}(t)-N^{2}(t)\right) d t^{2}+2 N_{\alpha}(t) \sigma_{i}^{\alpha}(x) d t d x^{i} \\
& +\gamma_{\alpha \beta}(t) \sigma_{i}^{\alpha}(x) \sigma_{j}^{\beta}(x) d x^{i} d x^{j}
\end{aligned}
$$

With Equation (3.2):

$\sigma_{\mathrm{i}, \mathrm{j}}^{\alpha}(\mathrm{x})-\sigma_{\mathrm{j}, \mathrm{i}}^{\alpha}(\mathrm{x})=2 \mathrm{C}_{\mu \mathrm{v}}^{\alpha} \sigma_{\mathrm{i}}^{\mathrm{v}}(\mathrm{x}) \sigma_{\mathrm{j}}^{\mu}(\mathrm{x})$

where, $\gamma_{\alpha \beta}(t)$ is the metric induced on the surfaces $\Sigma_{t}$ (and constant on them), $\mathrm{N}(\mathrm{t})$ is the lapse function, $\mathrm{N}_{\alpha}(\mathrm{t})$ is the shift vector $\left(\mathrm{N}^{\alpha}(\mathrm{t})=\gamma^{\alpha \beta}(\mathrm{t}) \mathrm{N}_{\beta}(\mathrm{t}), \gamma^{\alpha \beta}(\mathrm{t})\right.$ being the inverse of $\left.\gamma_{\alpha \beta}(t)\right)$ and $C_{\mu v}^{\alpha}$ are the structure constants for the corresponding Lie Algebra. Greek indices count the difierent one-forms, while the Latin are world indices; both kind of indices range in the interval (Isham, 1995; Sundermeyer, 1982). In 2 dimensions, there are only two distinct Lie Algebras; the Abelian(I), where all the structure constant vanish $C_{\mu v}^{\alpha}=0$ and the Non Abelian (II), where there is only one independent non vanishing structure constant-say $C_{12}^{1}=1$; every other choice for the non vanishing structure constants can be transformed into this, under a linear mixing of the initial set i.e., using a new set of the form $\tilde{C}_{\mu v}^{\alpha}=\Upsilon_{\beta}^{\alpha} \Delta_{\mu}^{k} \Delta_{v}^{\lambda} C_{k \lambda}^{\beta}$ where $\Delta_{\beta}^{\alpha} \in \mathrm{GL}(2, \mathbb{R})$ and $\Upsilon_{\beta}^{\alpha} \quad$ its inverse. Thus, two "cosmological" models emerge for the pure gravity case and two more when a $\Lambda$ term is present.

At this point, a question arises; is there any particular class of General Coordinate Transformations (G.C.T.'s) which can serve to simplify the form of Einstein's Field Equations (E.F.E.'s)? The answer is positive and a thorough investigation of this problem and its consequences, is given in (Christodoulakis et al., 2001); indeed, not only there is a class of G.C.T.'s which 
preserve the manifest spatial homogeneity of the line element (3.1), but also forms a continuous (and virtually, Lie) group. This group is closely related to the symmetries of the symmetry Lie Group $\mathrm{G}_{\mathrm{r}}$; it is its auto orphism group. A brief account of the relevant findings of (Christodoulakis et al., 2001) is as follows:

Consider the transformations Equation (3.3a and $b$ ):

$$
\begin{aligned}
& t \rightarrow \tilde{t}=t \Leftrightarrow t=\tilde{t} \\
& x^{i} \rightarrow \tilde{x}^{i}=g^{i}\left(t, x^{j}\right) \Leftrightarrow x^{i}=f^{i}\left(t, \tilde{x}^{j}\right)
\end{aligned}
$$

When we insert (3.3) into (3.1), the need to preserve the manifest spatial homogeneity of the latter, leads to the restrictions Equation (3.4a and $\mathrm{b}$ ):

$$
\begin{aligned}
& \frac{\partial \mathrm{f}^{\mathrm{i}}}{\partial \mathrm{t}}=\sigma_{\alpha}^{\mathrm{i}}(\mathrm{f}) \mathrm{P}^{\alpha}(\mathrm{t}) \\
& \frac{\partial \mathrm{f}^{\mathrm{i}}}{\partial \tilde{\mathrm{x}}^{\mathrm{j}}}=\sigma_{\alpha}^{\mathrm{i}}(\mathrm{f}) \Lambda_{\beta}^{\alpha}(\mathrm{t}) \sigma_{\mathrm{j}}^{\beta}(\tilde{\mathrm{x}})
\end{aligned}
$$

And, subsequently, to the identifications Equation (3.5a to c):

$$
\begin{aligned}
& \tilde{N}(t)=N(t) \\
& \tilde{N}^{\alpha}(t)=S_{\beta}^{\alpha}(t)\left(N^{\beta}(t)+P^{\beta}(t)\right) \\
& \tilde{\gamma}_{\alpha \beta}(t)=\Lambda_{\alpha}^{\mu}(t) \Lambda_{\beta}^{v}(t) \gamma_{\mu v}(t)
\end{aligned}
$$

where, $\mathrm{N}^{\alpha}(\mathrm{t})=\gamma^{\alpha \beta}(\mathrm{t}) \mathrm{N}_{\beta}\left(\mathrm{t}\right.$, ), with $\sigma_{\alpha}^{\mathrm{i}}(\mathrm{x})$ being the matrix inverse to $\sigma_{\mathrm{i}}^{\alpha}(\mathrm{x})$ Integrability conditions for the system (3.4) i.e., Frobenious' Theorem, results in the system (the dot, whenever used, denotes difierentiation with respect to time) Equation (3.6a and $b$ ):

$$
\begin{aligned}
& \mathrm{C}_{\mu v}^{\beta} \Lambda_{\beta}^{\alpha}(\mathrm{t})=\mathrm{C}_{\kappa \lambda}^{\alpha} \Lambda_{\mu}^{\mathrm{k}}(\mathrm{t}) \Lambda_{\mathrm{v}}^{\lambda}(\mathrm{t}) \\
& \frac{1}{2} \dot{\Lambda}_{\beta}^{\alpha}(\mathrm{t})=\mathrm{C}_{\mu \nu}^{\alpha} \mathrm{P}^{\mu}(\mathrm{t}) \Lambda_{\beta}^{\mathrm{v}}(\mathrm{t})
\end{aligned}
$$

And, “Time-Dependent Automorphisms Inducing Difieomorphisms" emerge. The automorphisms of a Lie Group $G_{r}$ constitute a continuous group. The members of the group which are continuously connected to the identity element, form a Lie Group as well-though the topology of the latter might be difierent from that of the former. If one considers parametric families of the automorphic matrices, characterized by the parameters $\tau^{\mathrm{i}}, \Lambda_{\beta}^{\alpha}\left(\mathrm{t} ; \tau^{\mathrm{i}}\right)$ and, as usual, demands Equation (3.7a and b):

$$
\begin{aligned}
& \left.\Lambda_{\beta}^{\alpha}\left(\mathrm{t} ; \tau^{\mathrm{i}}\right)\right|_{\tau^{\mathrm{i}}=0}=\delta_{\beta}^{\alpha} \\
& \left.\frac{\mathrm{d} \Lambda_{\beta}^{\alpha}\left(\mathrm{t} ; \tau^{\mathrm{i}}\right)}{\mathrm{d} \tau^{\mathrm{i}}}\right|_{\tau^{j \neq i}=0}=\lambda_{\beta(\mathrm{i})}^{\alpha}
\end{aligned}
$$

where, $\lambda_{\beta(i)}^{\alpha}$, are the generators with respect to the parameter $\tau^{\mathrm{i}}$, of the Lie Algebra of the Automorphisms, then from the first of (3.6), after a difierentiation with respect to $\tau^{\mathrm{i}}$, will have Equation (3.8):

$\lambda_{\beta(\mathrm{i})}^{\alpha} \mathrm{C}_{\mu v}^{\beta}=\lambda_{\mu(\mathrm{i})}^{\rho} \mathrm{C}_{\rho v}^{\alpha}+\lambda_{v(\mathrm{i})}^{\rho} \mathrm{C}_{\mu \rho}^{\alpha}$

For an extensive treatment on these issues see (Christodoulakis et al., 2002a), while for the relation and usage of these generators with conditional symmetries (Kuchar, 1982; Christodoulakis et al., 2002b).

In $n+1$ analysis (here $n=2$ ), the E.F.E.'s in vacuum, when the cosmological constant $\Lambda_{\mathrm{c}}$ exists, take the form Equation (3.9a to c):

$$
\begin{aligned}
& \mathrm{E}_{0}^{0}=\mathrm{K}_{\beta}^{\alpha} \mathrm{K}_{\alpha}^{\beta}-\mathrm{K}^{2}+\left(\mathrm{R}+2 \Lambda_{\mathrm{c}}\right)=0 \\
& \mathrm{E}_{\alpha}^{0}=\mathrm{K}_{v}^{\mu} \mathrm{C}_{\alpha \mu}^{v}-\mathrm{K}_{\alpha}^{\mu} C_{\mu v}^{v}=0
\end{aligned}
$$

$$
\begin{aligned}
& \mathrm{E}_{\beta}^{\alpha}=\dot{\mathrm{K}}_{\beta}^{\alpha}-\mathrm{NKK}_{\beta}^{\alpha}+\mathrm{N}\left(\mathrm{R}_{\beta}^{\alpha}+\frac{2}{\mathrm{n}-1} \Lambda_{\mathrm{c}} \delta_{\beta}^{\alpha}\right) \\
& +2 \mathrm{~N}^{\rho}\left(\mathrm{K}_{v}^{\alpha} \mathrm{C}_{\beta \rho}^{v}-\mathrm{K}_{\beta}^{v} \mathrm{C}_{v \rho}^{\alpha}\right)=0
\end{aligned}
$$

With Equation (3.10a to d):

$$
\begin{aligned}
& \mathrm{K}_{\beta}^{\alpha}(\mathrm{t})=\gamma^{\alpha \rho}(\mathrm{t}) \mathrm{K}_{\rho \beta}(\mathrm{t}) \\
& \mathrm{R}_{\beta}^{\alpha}(\mathrm{t})=\gamma^{\alpha \rho}(\mathrm{t}) \mathrm{R}_{\rho \beta}(\mathrm{t}) \\
& \mathrm{K}_{\alpha \beta}(\mathrm{t})=-\frac{1}{2 \mathrm{~N}(\mathrm{t})}\left(\begin{array}{l}
\dot{\gamma}_{\alpha \beta}(\mathrm{t})+2 \gamma_{\alpha v}(\mathrm{t}) \mathrm{C}_{\beta \rho}^{v} \mathrm{~N}^{\rho}(\mathrm{t}) \\
+2 \gamma_{\beta v}(\mathrm{t}) \mathrm{C}_{\alpha \rho}^{v} \mathrm{~N}^{\rho}(\mathrm{t})
\end{array}\right)
\end{aligned}
$$


$\mathrm{R}_{\alpha \beta}(\mathrm{t})=\mathrm{C}_{\sigma \tau}^{\mathrm{k}} \mathrm{C}_{\mu v}^{\lambda} \gamma_{\alpha \kappa}(\mathrm{t}) \gamma_{\beta \lambda}(\mathrm{t}) \gamma^{\sigma v}(\mathrm{t}) \gamma^{\tau u}(\mathrm{t})$

$+2 \mathrm{C}_{\alpha \mathrm{k}}^{\lambda} \mathrm{C}_{\beta \lambda}^{\mathrm{k}}+2 \mathrm{C}_{\alpha \mathrm{k}}^{\mu} \mathrm{C}_{\beta \lambda}^{\mathrm{v}} \gamma_{\mu \nu}(\mathrm{t}) \gamma^{\mathrm{k \lambda}}(\mathrm{t})$

$+2 \mathrm{C}_{\alpha \mathrm{K}}^{\lambda} \mathrm{C}_{\mu v}^{\mu} \gamma_{\beta \lambda}(\mathrm{t}) \gamma^{\mathrm{kv}}(\mathrm{t})+2 \mathrm{C}_{\beta \mathrm{K}} \mathrm{C}_{\mu \nu}^{\mu} \Upsilon_{\alpha \lambda}(\mathrm{t}) \Upsilon^{\mathrm{kv}}(\mathrm{t})$

where, $\delta_{\beta}^{\alpha}$ is the Kronecker's Delta.

Since G.C.T.'s , in general, are covariances of the E.F.E.'s, the same must hold true for the Time Dependent A.I.D.'s. Indeed, under the "gauge" transformations (3.5) one can readily proof (with the help of 3:6) Equation (3.11a to c):

$$
\tilde{\mathrm{E}}_{0}^{0}=\mathrm{E}_{0}^{0}
$$

$\tilde{\mathrm{E}}_{\alpha}^{0}=\Lambda_{\alpha}^{\beta} \mathrm{E}_{\beta}^{0}$

$\tilde{\mathrm{E}}_{\beta}^{\alpha}=\mathrm{S}_{\kappa}^{\alpha} \Lambda_{\beta}^{\lambda} \mathrm{E}_{\lambda}^{\kappa}$

where, $S_{\beta}^{\alpha}$ is the matrix inverse to $\Lambda_{\beta}^{\alpha}$. The effect of a time reparameterization can also be trivially seen to be a covariance of (3.9). Using the freedom contained in $\mathrm{P}^{\alpha}(\mathrm{t})$ one can always set the shift to zero which, together with the choice of time $\mathrm{N}(\mathrm{t})=\sqrt{\gamma(\mathrm{t})}$ considerably simplifies the E.F.E.'s (3.9) which become Equation (3.12a to c):

$$
\begin{aligned}
& -\gamma^{\alpha \mu} \gamma^{\beta v} \dot{\gamma}_{\alpha \beta} \dot{\gamma}_{\mu v}+\left(\frac{\dot{\gamma}}{\gamma}\right)^{2}-4 \gamma\left(\mathrm{R}+2 \Lambda_{\mathrm{c}}\right)=0 \\
& \gamma^{\mu \rho} \dot{\gamma}_{\rho v} \mathrm{C}_{\alpha \mu}^{\mathrm{v}}-\gamma^{\mu \rho} \dot{\gamma}_{\rho \alpha} \mathrm{C}_{\mu v}^{\mathrm{v}}=0 \\
& \left(\gamma^{\alpha \rho} \dot{\gamma}_{\beta \rho}\right)-2 \gamma\left(\mathrm{R}_{\beta}^{\alpha}+2 \Lambda_{\mathrm{c}} \delta_{\beta}^{\alpha}\right)=0
\end{aligned}
$$

where, tildes have been omitted. Finally some terminology may prove helpful; (3.9a) is called Quadratic Constraint, (3.9b) are called Linear Constraints and (3.9c) are simply the Equations of Motion.

\section{THE ABELIAN MODEL-CLASSICAL CONSIDERATION}

This model is characterized by the vanishing of all the structure constants of the corresponding

Lie Algebra. In particular, since $C_{\mu \alpha}^{\alpha}=0$, it is also a Class A model. A choice for the basis one-forms, in the spatial coordinate basis $(\mathrm{x}, \mathrm{y})$, is $\sigma_{\mathrm{i}}^{\alpha}(\mathrm{x})=\delta_{i}^{\alpha}$ i.e., the Kronecker's Delta. In matrix notation Equation (4.1):

$$
\sigma_{\mathrm{i}}^{\alpha}(\mathrm{x})=\left(\begin{array}{ll}
1 & 0 \\
0 & 1
\end{array}\right)
$$

The solution to the system (3.6) is Equation (4.2a and $b$ ):

$$
\begin{aligned}
& \Lambda_{\beta}^{\alpha} \in \mathrm{GL}(2, \mathbb{R}) \\
& \mathrm{P}^{\alpha}(\mathrm{t})=(\mathrm{x}(\mathrm{t}), \mathrm{y}(\mathrm{t}))
\end{aligned}
$$

A suitable basis, which spans the space of solutions to (3.8) is Equation (4.3):

$$
\begin{aligned}
& \lambda_{\beta(1)}^{\alpha}=\left(\begin{array}{cc}
1 & 0 \\
0 & -1
\end{array}\right) \lambda_{\beta(2)}^{\alpha}=\left(\begin{array}{ll}
0 & 1 \\
0 & 0
\end{array}\right) \lambda_{\beta(3)}^{\alpha} \\
& =\left(\begin{array}{ll}
1 & 0 \\
0 & 1
\end{array}\right) \lambda_{\beta(4)}^{\alpha}=\left(\begin{array}{ll}
0 & 0 \\
1 & 0
\end{array}\right)
\end{aligned}
$$

It is noteworthy that $\mathrm{P}^{\alpha}(\mathrm{t})$ contains the entire freedom carried by the Time Dependent A.I.D.'s, i.e., the two arbitrary functions of time. Thus, since the matrix $\Lambda_{\beta}^{\alpha}$ is constant, the only possible use of this "gauge" freedom is, according to (3.5), to set the shift zero. Choosing the above mentioned time gauge, we have Equation (4.4a to c):

$-\gamma^{\alpha \mu} \gamma^{\beta \nu} \dot{\gamma}_{\alpha \beta} \dot{\gamma}_{\mu v}+\left(\frac{\dot{\gamma}}{\gamma}\right)^{2}-8 \gamma \Lambda_{c}=0$

Linear Constraints are identically satisftied

$$
\left(\gamma^{\alpha \rho} \dot{\gamma}_{\beta \rho}\right)-4 \gamma \Lambda_{c} \delta_{\beta}^{\alpha}=0
$$

\subsection{Abelian Model with Vanishing Cosmological Constant}

The previous set of equations assumes the form Equation (4.5a to c):

$\left(\vartheta_{\alpha}^{\alpha}\right)^{2}-\vartheta_{\beta}^{\alpha} \vartheta_{\alpha}^{\beta}=0$

Linear Constraints are Identically Satised

$\gamma^{\alpha \omega} \dot{\gamma}_{\omega \beta}-\vartheta_{\beta}^{\alpha}=0$

where, $\vartheta_{\beta}^{\alpha}$ is a constant matrix. The integration of (4.5) is straightforward. The quadratic constraint, simply states that 
the determinant of the matrix $\vartheta_{\beta}^{\alpha}$ vanishes; thus the initial number of the independent components, is at most 3. But the equations of motion, can be rewritten as Equation (4.6):

$\dot{\gamma}_{\alpha \beta}=\gamma_{\alpha \mathrm{e}} \vartheta_{\beta}^{\mathrm{e}}$

And a consistency requirement, emerges Equation (4.7):

$$
\dot{\gamma}_{\alpha \beta}=\dot{\gamma}_{\beta \alpha} \Rightarrow \gamma_{\alpha \mathrm{e}} \vartheta_{\beta}^{\mathrm{e}}=\gamma_{\beta \mathrm{e}} \vartheta_{\alpha}^{\mathrm{e}}
$$

An exhaustive consideration of all the cases concerning a matrix $\vartheta_{\beta}^{\alpha}$, with vanishing determinant, plus the consistency requirement, results in only the following two distinct-at first sight-solutions to the previous system Equation (4.8):

$$
\gamma_{\alpha \beta}=\left(\begin{array}{ll}
1 & 0 \\
0 & 1
\end{array}\right)
$$

with associated line element Equation (4.9 and 4.10):

$$
\left(\mathrm{ds}_{1}\right)^{2}=-(\mathrm{dt})^{2}+\left(\mathrm{dx}^{1}\right)^{2}+\left(\mathrm{dx}^{2}\right)^{2}
$$

And:

$\gamma_{\alpha \beta}(\tau)=\left(\begin{array}{cc}1 & 0 \\ 0 & \mathrm{e}^{\theta \tau}\end{array}\right), \theta \in \mathbb{R}^{*}$

where, $\mathbb{R}^{*}=\mathbb{R}-[0]$, with associated line element Equation (4.11):

$$
\left(\mathrm{ds}_{2}\right)^{2}=-\mathrm{e}^{\theta \tau}(\mathrm{d} \tau)^{2}+\left(d y^{1}\right)^{2}+\mathrm{e}^{\theta \tau}\left(\mathrm{dy}^{2}\right)^{2}, \theta \in \mathbb{R}^{*}
$$

The transformation $\left(t, x^{1}, x^{2}\right) \rightarrow\left(\tau, y^{1}, y^{2}\right)$ Equation (4.12):

$$
\left(\mathrm{t}, \mathrm{x}^{1}, \mathrm{x}^{2}\right)=\left(\frac{2}{\theta} \cosh \left(\frac{\theta \mathrm{y}^{2}}{2}\right) \mathrm{e}^{\theta \tau / 2}, \mathrm{y}^{1}, \frac{2}{\theta} \sinh \left(\frac{\theta \mathrm{y}^{2}}{2}\right) \mathrm{e}^{\theta \tau / 2}\right)
$$

Takes the line element $\left(\mathrm{ds}_{1}\right)^{2}$ to $\left(\mathrm{ds}_{2}\right)^{2}$.

\subsection{Abelian Model with Non Vanishing Cosmological Constant}

Contraction of the equations of motion results in the integral of motion Equation (4.13): $\left(\frac{\dot{\gamma}}{\gamma}\right)^{2}-16 \gamma \Lambda_{c}=2 \omega=$ const

Under the "conformal" change of depended variable $\gamma_{\alpha \beta}=\bar{\gamma}_{\alpha \beta} \sqrt{\gamma}$ the equations of motion become Equation (4.14):

$\bar{\gamma}^{\alpha \omega} \dot{\bar{\gamma}}_{\omega \beta}-\vartheta_{\beta}^{\alpha}=0$

And contraction of this set, results in ( $\sin c e \bar{\gamma}=1) \vartheta_{\alpha}^{\alpha}=0$. Substitution of the quadratic constraint and usage of the integral of motion (4.13), yield Equation (4.15):

$\omega=\vartheta_{\beta}^{\alpha} \vartheta_{\alpha}^{\beta} \Rightarrow \omega=2 \vartheta_{1}^{1}+\vartheta_{2}^{1} \vartheta_{1}^{2}$

Since $\vartheta_{\alpha}^{\alpha}=0$.

Again, consideration of the equations of motion in terms of $\bar{\gamma}_{\alpha \beta}$ for all the traceless matrices $\vartheta_{\alpha}^{\alpha}$, plus the consistency requirement (i.e., $\dot{\bar{\gamma}}_{\alpha \beta}=\dot{\bar{\gamma}}_{\beta \alpha}$ ) produce the following two solutions Equation (4.16 and 4.17):

$\bar{\gamma}_{\alpha \beta}=\left(\begin{array}{ll}1 & 0 \\ 0 & 1\end{array}\right)$, with $\omega=0$

And:

$\bar{\gamma}_{\alpha \beta}(\tau)=\left(\begin{array}{cc}\mathrm{e}^{\vartheta_{1}^{\prime} \tau} & 0 \\ 0 & \mathrm{e}^{-\vartheta_{1}^{\prime} \tau}\end{array}\right)$, with $\omega=2\left(\vartheta_{1}^{1}\right)^{2}>0, \vartheta_{1}^{1} \in \mathbb{R}^{*}$

Since, for all the cases, $\omega \geq 0$ the integral of motion (4.13) dictates that $\Lambda_{c}>0$. Inversion of the conformal transformation and integration of (4.13) for each of the two solutions, yield Equation (4.18):

$\gamma_{\alpha \beta}(t)=\frac{1}{2 t \sqrt{\Lambda_{c}}}\left(\begin{array}{ll}1 & 0 \\ 0 & 1\end{array}\right)$

With associated line element Equation (4.19):

$\left(\mathrm{ds}_{1}\right)^{2}=-\frac{1}{4 \mathrm{t}^{2} \Lambda_{\mathrm{c}}}(\mathrm{dt})^{2}+\frac{1}{2 \mathrm{t} \sqrt{\Lambda_{\mathrm{c}}}}\left(\left(\mathrm{dx}^{1}\right)^{2}+\left(\mathrm{dx}^{2}\right)^{2}\right)$

And Equation (4.20): 


$$
\gamma_{\alpha \beta}(\tau)=\frac{\vartheta_{1}^{1}}{2 \sqrt{\Lambda_{c}}}\left(\begin{array}{cc}
e^{\vartheta_{1}^{1} \tau} & 0 \\
0 & \mathrm{e}^{-\vartheta_{1}^{1} \tau}
\end{array}\right), \vartheta_{1}^{1} \in \mathbb{R}^{*}
$$

With associated line element Equation (4.21):

$$
\begin{aligned}
& \left(\mathrm{ds}_{2}\right)^{2}=-\frac{1}{4 \Lambda_{\mathrm{c}} \sinh ^{2}(\tau)}(\mathrm{d} \tau)^{2}+\frac{\mathrm{e}^{\tau}}{2 \sqrt{\Lambda_{\mathrm{c}}} \sinh (\tau)}\left(\mathrm{dy}^{1}\right)^{2} \\
& +\frac{\mathrm{e}^{-\tau}}{2 \sqrt{\Lambda_{\mathrm{c}}} \sinh (\tau)}\left(\mathrm{dy}^{2}\right)^{2}
\end{aligned}
$$

where, $\vartheta_{1}^{1}$, not being an essential constant (Christodoulakis et al., 2001), has been absorbed.

It is obvious that the two line elements are connected by a time reparameterization of the form $t \rightarrow \tau: t=\sinh (\tau) \mathrm{e}^{-\tau}$.

\section{THE NON ABELIAN MODEL- CLASSICAL CONSIDERATION}

This model is characterized by $C_{12}^{1}=1$; any other choice can be cast into this. A choice for the basis one-forms, in the spatial coordinate basis $\left(\mathrm{x}^{1}, \mathrm{x}^{2}\right)$ is Equation (5.1):

$$
\sigma_{i}^{\alpha}(x)=\left(\begin{array}{cc}
e^{-2 x^{2}} & 0 \\
0 & 1
\end{array}\right)
$$

Now, the solution to the system (3.6) is Equation (5.1a and $\mathrm{b})$ :

$$
\begin{aligned}
& \Lambda_{\beta}^{\alpha}(t)=\left(\begin{array}{cc}
x(t) & y(t) \\
0 & 1
\end{array}\right) \\
& P^{\alpha}(t)=\left(\frac{\dot{y}(t)}{2}-\frac{y(t) \dot{x}(t)}{2 x(t)},-\frac{\dot{x}(t)}{2 x(t)}\right)
\end{aligned}
$$

This time, both the automorphic matrices $\Lambda_{\beta}^{\alpha}(\mathrm{t})$ and the triplet $\mathrm{P}^{\alpha}(\mathrm{t})$ carry the freedom contained in the time dependent A.I.D.'s. It is wiser to exploit this freedom contained in $\Lambda_{\beta}^{\alpha}(\mathrm{t})$ in order to simplify the initial form of the scale factor matrix $\gamma_{\alpha \beta}(\mathrm{t})$ rather than use the same freedom contained in $\mathrm{P}^{\alpha}(\mathrm{t})$ to set the shift vector equal to zero. Accordingly, an initial full scale factor matrix, can be brought to the form Equation (5.3):

$$
\gamma_{\alpha \beta}(t)=\left(\begin{array}{cc}
\gamma_{11}(t) & 0 \\
0 & \gamma_{11}(t)
\end{array}\right)
$$

Thus the initial set of "dynamical" variables, consists of a scale factor matrix of the previous form, plus a shift vector $\left(\mathrm{N}^{1}(\mathrm{t}), \mathrm{N}^{2}(\mathrm{t})\right)$. Insertion of this set into both (3.9b) and (3.10d) results in respectively Equation (5.4 and 5.5):

$$
\mathrm{N}^{\alpha}(\mathrm{t})=0
$$

And:

$$
\mathrm{R}_{\beta}^{\alpha}=\frac{4}{\gamma_{11}(\mathrm{t})}\left(\begin{array}{ll}
1 & 0 \\
0 & 1
\end{array}\right)
$$

Thus, a judicious use of the gauge freedom can result in both a very simple form of the scale factor matrix and the vanishing of the shift. The equations of motion admit the integral of motion Equation (5.6):

$$
\left(\frac{\dot{\gamma}_{11}}{\gamma_{11}}\right)^{2}-\left(16 \gamma_{11}+4 \Lambda_{c} \gamma_{11}^{2}\right)=\omega=\text { const }
$$

The quadratic constraint assumes the form Equation (5.7):

$\left(\frac{\dot{\gamma}_{11}}{\gamma_{11}}\right)^{2}-\left(16 \gamma_{11}+4 \Lambda_{c} \gamma_{11}^{2}\right)=0$

and thus not only sets the constant $\mathrm{w}$ equal to zero, but also demands $\Lambda_{\mathrm{c}}>0$-if this term exists. The integration of (5.6) with $\omega=0$, is a trivial matter:

When $\Lambda_{\mathrm{c}}=0$ the result is Equation (5.8):

$$
\gamma_{11}(t)=\frac{1}{4 t^{2}}
$$

with corresponding line element Equation (5.9):

$$
\left(d s^{2}\right)=-\frac{1}{16 t^{2}}\left(d t^{2}\right)+\frac{e^{-4 x^{2}}}{4 t^{2}}\left(d x^{1}\right)^{2}+\frac{1}{4 t^{2}}\left(d x^{2}\right)^{2} t \in \mathbb{R}^{*}
$$

The transformation $\left(t, x^{1}, x^{2}\right) \rightarrow(T, X, Y)$ Equation (5.10):

$$
(T, X, Y)=\left(\begin{array}{l}
\frac{\cosh \left(2 x^{2}\right)}{4 t}+2 \frac{\left(x^{1}\right)^{2} e^{-2 x^{2}}}{4 t}, \frac{\sinh \left(2 x^{2}\right)}{4 t} \\
+2 \frac{\left(x^{1}\right)^{2} e^{-2 x^{2}}}{4 t},-2 \frac{x^{1} e^{-2 x^{2}}}{4 t}
\end{array}\right)
$$


Takes the standard Minkowski spacetime form to the line element above.

When $\Lambda_{\mathrm{c}} 6=0$ the result reads Equation (5.11):

$$
\gamma_{11}(t)=\frac{4}{16 t^{2}-\Lambda_{c}}
$$

The associated line element being Equation (5.12):

$$
\begin{aligned}
& (\mathrm{ds})^{2}=-\left(\frac{4}{16 \mathrm{t}^{2}-\Lambda_{\mathrm{c}}}\right)^{2}(\mathrm{dt})^{2}+\frac{4 \mathrm{e}^{-4 \mathrm{x}^{2}}}{16 \mathrm{t}^{2}-\Lambda_{\mathrm{c}}}\left(\mathrm{dx}^{1}\right)^{2} \\
& +\frac{4}{16 \mathrm{t}^{2}-\Lambda_{\mathrm{c}}}\left(\mathrm{dx}^{2}\right)^{2} \mathrm{t} \in \mathbb{R}_{*}
\end{aligned}
$$

$$
\text { With } \mathbb{R}_{*}=\mathbb{R}-\left[-\sqrt{\Lambda_{\mathrm{c}}} / 4, \sqrt{\Lambda_{\mathrm{c}}} / 4\right]
$$

\section{QUANTUM DESCRIPTION OF The MODELS}

In trying to quantize gravity, one faces the problem of quantizing a constrained system. The main steps one has to follow are:

- Define the basic operators $\hat{g}_{\mathrm{ij}}$ and $\hat{\pi}^{\mathrm{ij}}$ and the canonical commutation relation they satisfy

- Define quantum operators $\hat{\mathrm{H}}_{\mathrm{m}}$ whose classical counterparts are the constraint functions $\mathrm{H}_{\mathrm{m}}$

- Define the quantum states $\Psi[\mathrm{g}]$ as the common null eigenvector of $\hat{\mathrm{H}}_{\mathrm{m}}$, i.e., those satisfying $\hat{\mathrm{H}}_{\mathrm{m}} \Psi[\mathrm{g}]=$ 0. (As a consequence, one has to check that $\hat{\mathrm{H}}_{\mathrm{m}}$, form a closed algebra under the basic Canonical Commutation Relations (CCR)

- Find the states and define the inner product in the space of these states.

Concerning point (iii) it is pertinent to clarify the meaning of the imposition of the quantum constraints upon $\Psi[\mathrm{g}]$. A straightforward (modulo regularization prescriptions) but tedious calculation shows that any functional which is not a scalar functional of the curvature and/or higher derivative curvature scalars does not solve the linear operator constraints. Therefore, the imposition of these conditions, ensures that the wave functional will be a (scalar) functional of the 3-geometry and not of the coordinate system on it. Then, the dynamical evolution is provided by the quadratic operator constraint; the consistency of the quantum algebra is, somehow, expected to guarantee that the final wave functional will be independent of the 4 dimensional coordinate system.

In the absence of a full solution to the problem, a partial solution, generally known as quantum cosmology, has been employed. This is an approximation to quantum gravity in which one freezes out all but a finite number of degrees of freedom and quantizes the rest. In this way one is left with a much more manageable problem that is essentially quantum mechanics with constraints. In principle, the dynamical variables are the components of a $2 \times 2$ symmetric scale factor matrix $\gamma_{\alpha \beta}(\mathrm{t})$ 's, the lapse function $\mathrm{N}(\mathrm{t})$ and the shift vector $\mathrm{N}^{\alpha}(\mathrm{t})$. The presence of the linear constraints-along with the conditional symmetries of the corresponding Hamiltonian-enable a reduction of the initial configuration space to a lower dimensional one, spanned by the curvature scalar characterizing the 2-geometry. The ultimate justification of this reduction is the fact that from the point of view of the 2-geometry-the omitted degrees of freedom, are not physical but gauge (Christodoulakis et al., 2002a). It is true that at the classical level, the scale factor matrix, can be diagonalized on mass-shell-through a constant matrix e.g., (Christodoulakis et al., 2001) for the 3dimensional analogous case-while the shift can be set equal to zero. However, if one intends to give weight to all states, one has to start with the most general form which is described by the 3 scale factors $\gamma_{\alpha \beta}(t)$ 's and the lapse function $\mathrm{N}(\mathrm{t})$. The absence of $\mathrm{H}_{\alpha}$ 's due to the vanishing of the $C_{\beta \gamma}^{\alpha}$ 's, implies that in principle all $\gamma_{\alpha \beta}$ 's are candidates as arguments for the wave function which solves the quadratic constraint (Wheeler-DeWitt equation). This is in contrast to what happens in the Non Abelian case, where one combination of $\gamma_{\alpha \beta}$ 's and $C_{\beta \gamma}^{\alpha}$ 's, parameterize the reduced configuration space.

\subsection{Quantization of the Abelian Model}

In this section, we present a complete reduction of the initial configuration space for the Abelian geometry-by extracting as many gauge degrees of freedom, as possible. Two separate cases are considered; when the cosmological constant is present and when is not. In either case, a wave function which depends on one degree of freedom is found, by imposing on it, the quantum versions of all classical integrals of motion as additional conditions.

The Hamiltonian of the a Class A, spatially homogeneous cosmological system is $\mathrm{H}=$ $\mathrm{N}(\mathrm{t}) \mathrm{H}_{0}+\mathrm{N}^{\alpha}(\mathrm{t}) \mathrm{H}_{\alpha}$, where Equation (6.1): 
$\mathrm{H}_{0}=\frac{1}{\sqrt[2]{\gamma}} \mathrm{L}_{\alpha \beta \mu v} \pi^{\alpha \beta} \pi^{\mu \nu}+\sqrt{\gamma}(\mathrm{R}+2 \Lambda)$

is the quadratic constraint, with Equation (6.2):

$\mathrm{L}_{\alpha \beta \mu v}=\gamma_{\alpha \mu} \gamma_{\beta v}+\gamma_{\alpha v} \gamma_{\beta \mu}-2_{\gamma \alpha} \beta \gamma_{\mu v}$

$\mathrm{R}=\mathrm{C}_{\lambda \mu}^{\beta} \mathrm{C}_{\theta \tau}^{\alpha} \gamma_{\alpha \beta} \gamma^{\theta \lambda} \gamma^{\tau \mu}+2 \mathrm{C}_{\beta \delta}^{\alpha} \mathrm{C}_{v \alpha}^{\delta} \gamma^{\beta v}+4 \mathrm{C}_{\mu \nu}^{\mu} \mathrm{C}_{\beta \lambda}^{\beta} \gamma^{\nu \lambda}$

$\gamma$ being the determinant of $\gamma_{\alpha \beta}$ and Equation (6.3):

$\mathrm{H}_{\alpha}=4 \mathrm{C}_{\alpha \rho}^{\mu} \gamma_{\beta \mu} \pi^{\beta \rho}$

Are the linear constraints. For all Class A Types, the canonical equations of motion, following from (6.1), are equivalent to Einstein's equations derived from line element-see (Sneddon, 1976) for the $3+1$ dimensional analogous.

The quantities $\mathrm{H}_{0}, \mathrm{H}_{\alpha}$ are weakly vanishing (Dirac, 1950), i.e. $\mathrm{H}_{0} \approx 0, \mathrm{H}_{\alpha} \approx 0$. For the Class $\mathrm{A}\left(\mathrm{C}_{\beta \gamma}^{\alpha}=0\right)$ Abelian Model, it can be seen \{using the basic Poisson Brackets Relations (PBR's) $\left\{\gamma_{\alpha \beta}, \pi^{\mu \mathrm{v}}\right\}=S_{\alpha \beta}^{\mu v}$-that these constraints are first class, obeying the following algebra Equation (6.4):

$\left\{\mathrm{H}_{0}, \mathrm{H}_{\alpha}\right\}=0$
$\left\{\mathrm{H}_{\alpha}, \mathrm{H}_{\beta}\right\}=-2 \mathrm{C}_{\alpha \beta}^{\gamma} \mathrm{H}_{\gamma}$,

Which ensures their preservation in time, i.e., $\dot{\mathrm{H}}_{0} \approx 0, \dot{\mathrm{H}}_{\alpha} \approx 0$ and establishes the consistency of the action.

If we follow Dirac's general proposal (Dirac, 1950) for quantizing this action, we have to turn $\mathrm{H}_{0}, \mathrm{H}_{\alpha}$, into operators annihilating the wave function $\Psi$.

In the Schrodinger representation Equation (6.5):

$$
\begin{aligned}
& \gamma_{\alpha \beta} \rightarrow \hat{\gamma}_{\alpha \beta}=\gamma_{\alpha \beta} \\
& \pi^{\alpha \beta} \rightarrow \hat{\pi}^{\alpha \beta}=-i \frac{\partial}{\partial_{\lambda \alpha \beta}},
\end{aligned}
$$

With the relevant operators, satisfying the basic Canonical Commutation Relations (CCR's) which correspond to the classical ones Equation (6.6):

$$
\left[\hat{\gamma}_{\alpha \beta}, \pi^{\mu \nu}\right]=i \delta_{\alpha \beta}^{\mu v}=\frac{i}{2}\left(\delta_{\alpha}^{\mu} \delta_{\beta}^{v}+\delta_{\beta}^{\mu} \delta_{\alpha}^{v}\right)
$$

In the Abelian case, $\mathrm{C}_{\mu v}^{\alpha}=0$, thus the only operator which must annihilate the wave function, is $\hat{\mathrm{H}}_{0}$; and the
Wheeler-DeWitt equation $\hat{\mathrm{H}}_{0} \Psi=0$, will produce a wave function, initially residing on a 3-dimensional configuration space-spanned by $\gamma_{\alpha \beta}$ 's. If the linear constraints existed, a first reduction of the initial configuration space, would be possible (Kuchar, 1982). New variables, instead of the 3 scale factors, would emerge-say qi, with $\mathrm{i}<3$. Then a new "physical" metric would be induced Equation (6.7):

$\mathrm{g}^{\mathrm{ij}}=\mathrm{L}_{\alpha \beta \mu \nu} \frac{\partial \mathrm{q}^{\mathrm{i}}}{\partial \gamma_{\alpha \beta}} \frac{\partial \mathrm{q}^{\mathrm{j}}}{\partial \gamma_{\mu \nu}}$

According to Kuchar's and Hajicek's (Kuchar, 1982) prescription, the "kinetic" part of $\mathrm{H}_{0}$ would have to be realized as the conformal Laplacian (in order for the equation to respect the conformal covariance of the classical action), based on the physical metric (6.7). In the presence of conditional symmetries, further reduction can take place, a new physical metric would then be defined similarly and the above mentioned prescription, would have to be used after the final reduction (Hájíček and Kuchař, 1990).

The Abelian case, is an extreme example in which all the linear constraints, vanish identically; thus no initial physical metric, exists -another peculiarity reecting the high spatial symmetry of the model under consideration. In compensation, a lot of integrals of motion exist ant the problem of reduction, finds its solution through the notion of "Conditional Symmetries". These linear in momenta integrals of motion, if seen as vector field on the configuration space spanned by $\gamma_{\alpha \beta}$ 's, induce through their integral curvesmotions of the form $\tilde{\gamma}_{\alpha \beta}=\Lambda_{\alpha}^{\mu} \Lambda_{\beta}^{v} \gamma_{\mu v}, \Lambda \in \mathrm{GL}(2, \mathbb{R})$ (section 2 of Christodoulakis et al., (2002a)) which not only are identical to the action of spatial difieomorphisms, but also describe the action of the automorphism group-since $\mathrm{GL}(2, \mathrm{R})$ is the $\operatorname{Aut}(\mathrm{G})$ which corresponds to the Abelian models.

The generators of this automorphism group, are (in a collective form and matrix notation) the following 4-one for each parameter Equation (6.8):

$\lambda_{(\mathrm{I}) \beta}^{\alpha}=\left(\begin{array}{cc}\alpha & \beta \\ \delta & \varepsilon\end{array}\right), I \in\{1, \ldots, 4\}$

With the defining property Equation (6.9):

$\mathrm{C}_{\mu v}^{\alpha} \lambda_{\alpha}^{\kappa}=\mathrm{C}_{\mu \sigma}^{\sigma} \lambda_{v}^{\sigma}+\mathrm{C}_{\sigma \mathrm{v}}^{\kappa} \lambda_{\mu}^{\sigma}$. 
Exponentiating all these matrices, one obtains the outer automorphism group of the Abelian model, since there is not Inner Automorphism subgroup (all structure constants vanish).

For full pure gravity, Hájíček and Kuchař (1990) has shown that there are no other first-class functions, homogeneous and linear in the momenta, except the linear constraints-ditto in $2+1$ analysis. If however, we impose extra symmetries, such quantities may emergeas it will be shown. We are therefore-according to Dirac (Dirac, 1950) -justified to seek the generators of these extra symmetries; their quantum-operator analogues will be imposed as additional conditions on the wave function. The justification for such an action, is obvious since these generators correspond to spatial diffeomorphisms-which are the covariance of the theory. Thus, these additional conditions are expected to lead us to the final reduction, by revealing the true degrees of freedom. Such quantities are, generally, called in the literature "Conditional Symmetries" (Hájíček and Kuchař 1990).

From matrices (6.8), we can construct the linear-in momenta-quantities Equation (6.10):

$\mathrm{E}_{(\mathrm{I})}=\lambda_{(1) \beta}^{\alpha} \gamma_{\alpha \rho} \pi^{\rho \beta}, \mathrm{I} \in\{1, \ldots, 4\}$

In order to write analytically these quantities, the following base is chosen Equation (6.11):

$\lambda_{1}=\left(\begin{array}{cc}1 & 0 \\ 0 & -1\end{array}\right), \lambda_{2}=\left(\begin{array}{ll}0 & 1 \\ 0 & 0\end{array}\right), \lambda_{3}=\left(\begin{array}{ll}0 & 0 \\ 1 & 0\end{array}\right), \lambda_{4}=\left(\begin{array}{ll}1 & 0 \\ 0 & 1\end{array}\right)$

It is straightforward to calculate the Poisson Brackets between $\mathrm{E}_{(\mathrm{I})}$ and $\mathrm{H}_{0}$ Equation (6.12):

$\left\{\mathrm{E}_{(\mathrm{I})}, \mathrm{H}_{0}\right\}=-2 \Lambda \mathrm{N} \sqrt{\gamma} \lambda_{(1) \alpha}^{\alpha}$

But, it holds that Equation (6.13):

$\dot{\mathrm{E}}_{(\mathrm{I})}\left\{\mathrm{E}_{(\mathrm{I})}, \mathrm{H}_{0}\right\}=-2 \Lambda \mathrm{N} \sqrt{\gamma} \lambda_{(1) \alpha}^{\alpha}$

-The last equality emerging by virtue of (6.12). Thus Equation (6.14):

$$
\dot{\mathrm{E}}_{(\mathrm{I})}\left\{\mathrm{E}_{(\mathrm{I})}, \mathrm{H}_{0}\right\}=0 \Rightarrow \mathrm{E}_{(\mathrm{I})}=\mathrm{K}_{(\mathrm{I})}=\text { cons tan ts, } \mathrm{I} \in\{1,2,3\}
$$

We therefore conclude that, when the cosmological constants is non vanishing, only the first three $\mathrm{E}_{(\mathrm{I})}$, are firstclass and thus integrals of motion. If $\Lambda$ vanishes, all the four quantities, are first-class. Out of the three quantities $\mathrm{E}_{(\mathrm{I})}$, only two are (functionally) independent, if we allow for the coefficients of the linear combination to be functions of the $\gamma_{\alpha \beta}$ 's; if the coefficients are only allowed to be numbers, all three are (linearly) independent.

The algebra of $\mathrm{E}_{(\mathrm{I})}$ can be easily seen to be Equation (6.15):

$$
\left\{\mathrm{E}_{(\mathrm{I})}, \mathrm{E}_{(\mathrm{J})}\right\}=-\frac{1}{2} \mathrm{C}_{\mathrm{IJ}}^{\mathrm{M}} \mathrm{E}_{(\mathrm{M})}, \mathrm{I}, \mathrm{J}, \mathrm{M} \in\{1, \ldots, 4\}
$$

where, Equation (6.16):

$$
\left\{\lambda_{(\mathrm{I})}, \lambda_{(\mathrm{J})}\right\}=\mathrm{C}_{\mathrm{IJ}}^{\mathrm{M}} \lambda_{(\mathrm{M})}, \mathrm{I}, \mathrm{J}, \mathrm{M} \in\{1, \ldots, 4\}
$$

The square brackets denoting matrix commutation.

The non vanishing structure constants of the algebra (6.16), are found to be Equation (6.17):

$C_{12}^{2}=2 C_{13}^{3}=-2 C_{23}^{1}=1$

At this point, in order to achieve the desired reduction, we propose that the quantities $\mathrm{E}_{(\mathrm{I})}$-with $\mathrm{I} \in\left\{{ }_{1}, \ldots, 3\right\}$-must be promoted to operational conditions acting on the requested wave function $\Psi$-since they are first class quantities and thus integrals of motion (6.14). In the Schrodinger representation Equation (6.18):

$\widehat{\mathrm{E}}_{(\mathrm{I})} \Psi=-\mathrm{i} \lambda_{(\mathrm{I}) \alpha}^{\tau} \gamma_{\tau \beta} \frac{\partial \Psi}{\partial \gamma_{\alpha \beta}}=\mathrm{K}_{(\mathrm{I})} \Psi, \mathrm{I} \in\{1, \ldots, 3\}$

In general, systems of equations of this type, must satisfy consistency conditions decreed by the Frobenious Theorem Equation (6.19):

$$
\begin{aligned}
& \widehat{\mathrm{E}}_{(\mathrm{J})} \Psi=\mathrm{K}_{(\mathrm{J})} \Psi \Rightarrow \widehat{\mathrm{E}}_{(\mathrm{I})} \widehat{\mathrm{E}}_{(\mathrm{I})} \Psi=\mathrm{K}_{(\mathrm{I})} \mathrm{K}_{(\mathrm{J})} \Psi \\
& \widehat{\mathrm{E}}_{(\mathrm{I})} \Psi=\mathrm{K}_{(\mathrm{I})} \Psi \Rightarrow \hat{\mathrm{E}}_{(\mathrm{J})} \widehat{\mathrm{E}}_{(\mathrm{I})} \Psi=\mathrm{K}_{(\mathrm{J})} \mathrm{K}_{(\mathrm{I})} \Psi
\end{aligned}
$$

Subtraction of these two and usage of (6.15), results in Equation (6.20):

$$
\mathrm{K}_{\mathrm{IJ}}^{\mathrm{M}} \widehat{\mathrm{E}}_{(\mathrm{M})} \Psi=0 \Rightarrow \mathrm{C}_{\mathrm{IJ}}^{\mathrm{M}} \mathrm{K}_{(\mathrm{M})}=0
$$

This relation constitutes a selection rule for the numerical values of the integrals of motion. In view of the Lie Algebra (6.17), selection rule (6.20) sets $\mathrm{K}_{1}=\mathrm{K}_{2}$ $=\mathrm{K}_{3}=0$. This fact restores the action of the difieomorphisms as covariances of the quantum theory, 
in the sense that now, we have conditions of the form $\widehat{\mathrm{E}}_{(\mathrm{I})} \Psi=0$. On the contrary, if we also had $\mathrm{E}(4)$ (as is the case $\Lambda=0$ ) then $\mathrm{K} 4$ would remain arbitrary. With this outcome and using the method of characteristics (Carabedian, 1986), the system of the two functionally independent P.D.E.'s (6.18), can be integrated. The result is Equation (6.21):

$$
\Psi=\Psi(\gamma)
$$

i.e., an arbitrary function of $\gamma$-the determinant of the scale factor matrix. Note that the solution (6.21) would have not changed if another base for the generators had been chosen. However, such a choice would have had affected the form of the system (6.18).

The next step, is to construct the Wheeler-DeWitt equation which is to be solved by the wave function (6.21). The degree of freedom, is 1 ; the $\mathrm{q}=\gamma$. According to Kuchar's proposal (Kuchar, 1982), upon quantization, the kinetic part of Hamiltonian is to be realized as the conformal Beltrami operator-based on the induced physical metric \{according to (6.7), with $\mathrm{q}=\gamma$ Equation (6.22):

$$
\mathrm{g}^{11}=\mathrm{L}_{\alpha \beta \mu \nu} \frac{\partial \gamma}{\partial \gamma_{\alpha \beta}} \frac{\partial \gamma}{\partial \gamma_{\mu \nu}}=\mathrm{L}_{\alpha \beta \mu \nu} \gamma^{2} \gamma^{\alpha \beta} \gamma^{\mu \nu} \stackrel{\text { first of (6.2) }}{=}-4 \gamma^{2}
$$

In the Schrodinger representation Equation (6.23):

$\frac{1}{2} L_{\alpha \beta \mu v} \pi^{\alpha \beta} \pi^{\mu v} \rightarrow-\frac{1}{2} a_{c}^{2}$

where, Equation (6.24):

$$
\square_{\mathrm{c}}^{2}=\square^{2}=\frac{1}{\sqrt{\mathrm{g}_{11}}} \partial \gamma\left\{\sqrt{\mathrm{g}_{11}} \mathrm{~g}^{11} \partial \gamma\right\}
$$

Is the 1-dimensional Laplacian based on $\mathrm{g} 11\left(\mathrm{~g}^{11} \mathrm{~g}_{11}=\right.$ 1). Note that in 1-dimension the conformal group is totally contained in the G.C.T. group, in the sense that any conformal transformation of the metric cannot produce any change in the-trivial-geometry and is thus reachable by some G.C.T. Therefore, no extra term in needed in (6.24), as it can also formally be seen by taking the limit $n$ $=1, \mathrm{R}=0$ in the general definition Equation (6.25):

$$
\square_{c}^{2}=\square^{2}+\frac{(d-2)}{4(d-1)} R=\square^{2}
$$

$$
\text { Thus Equation (6.26): }
$$

$$
\mathrm{H}_{0} \rightarrow \widehat{\mathrm{H}}_{0}=2 \gamma \partial \gamma(\gamma \partial \gamma)+2 \Lambda \gamma
$$

So, the Wheeler-DeWitt equation -by virtue of (6.21), reads Equation (6.27):

$\widehat{\mathrm{H}}_{0} \Psi=\gamma^{2} \Psi^{\prime \prime}+\gamma \Psi^{\prime}+\gamma \Lambda \Psi=0$

The general solution to this equation, is Equation (6.28):

$$
\Psi(\gamma)=\mathrm{c}_{1} \mathrm{~J}_{0}(2 \sqrt{\gamma \Lambda})+\mathrm{c}_{2} \mathrm{Y}_{0}(2 \sqrt{\gamma \Lambda})
$$

where, $J_{n}$ and $Y_{n}$, are the Bessel Functions of the first and second kind respectively -both of zero order- and $c_{1}$, $c_{2}$, arbitrary constants. Some comments on this wave function. Indeed, at first sight, the fact that $\Psi$ depends only on one argument and particularly on $\gamma$, seems to point to some undesirable degeneracy regarding anisotropy; classically $\gamma$ can be gauged to et and thus it seems as though the anisotropy parameter does not enter $\Psi$ at all. If, however, we reflect thoroughly, we will realize that this objection rests strongly on a -not generally accepted-mingling of the classical notion of anisotropy and the interpretation of the wave function. Indeed if we adopt the interpretation that the wave function $\Psi$ (along with a suitable measure), is to give weight to all configurations parameterized by $\gamma_{\alpha \beta}$, then the anisotropic configuration will, in general, acquire different probabilities. The degeneracy occurs only between two different anisotropic configurations with the same determinant $\gamma$. In compensation the scheme proposed here, avoids the gauge degrees of freedom as much as possible. The final probabilistic interpretation must await the selection of a proper measure.

If the cosmological constant $\Lambda$ is zero, some changes will take place. The first concerns the obvious alteration to the potential in the Hamiltonian; it vanishes. This consequently, causes an alteration to the Poisson Bracket (6.12), which takes the form Equation (6.29):

$$
\left\{\mathrm{E}_{(\mathrm{I})}, \mathrm{H}_{0}\right\}=0, \mathrm{I} \in\{1, \ldots, 4\}
$$

while, (6.15) still holds. Thus in the present case, there are four integrals of motion-instead of three. Also, the P.D.E. system (6.18) consists of four members (instead of three), but now out of the four quantities $\mathrm{E}_{(\mathrm{I})}$, only three are functionally independent; the previous two, plus $\mathrm{E}_{(4)}$. Again, using the method of characteristics (Carabedian, 1986), the system of the three functionally 
independent P.D.E.'s (6.18), can be integrated. The result is Equation (6.30):

$$
\Psi=\mathrm{c}_{1} \gamma^{\mathrm{i} \mathrm{K}_{4} / 2}
$$

where, $\gamma$ is the determinant of the scale factor and $\mathrm{K} 4$, the remaining constant according to selection rule (6.20).

The fact that this wave function does not depend on any combination of $\gamma_{\alpha \beta}$ 's in an arbitrary manner (i.e. $\Psi$ is not an arbitrary function of $\gamma_{\alpha \beta}$ 's), might be taken as an indication that no reduced Wheeler-DeWitt equation can be written. On the other hand, this wave function does contain an arbitrary constant which, at the classical level is not essential (the model is $2+1$ Minkowski spacetime). Thus, it would not be harmful if the value of this constant were to be fixed by the quantum dynamics. These thoughts lead to the following compromise; the initial configuration space, should be the minisuperspace i.e., we should write the Wheeler-DeWitt equation, based on the supermetric $\mathrm{L}^{\alpha \beta \mu \mathrm{v}}$.

In the Schrodinger representation Equation (6.31):

$$
\frac{1}{2} \mathrm{~L}_{\alpha \beta \mu v} \pi^{\alpha \beta} \pi^{\mu v} \rightarrow-\frac{1}{2} \square_{c}^{2}
$$

Thus using (A.11), (A.13), (A.14) for $\mathrm{n}=2$ and D $=3$, one may find respectively-see appendix Equation (6.32 and 6.33):

$$
\mathrm{R}=2
$$

$$
\mathrm{L}_{\alpha \beta \mu v} \Gamma_{\mathrm{k \lambda}}^{\alpha \beta \mu v}=-\gamma_{\mathrm{k} \lambda}
$$

And Equation (6.34):

$$
\square_{c}^{2}=\mathrm{L}_{\alpha \beta \mu v} \frac{\partial}{\partial \gamma_{\alpha \beta} \gamma_{\mu v}}+\gamma_{\mathrm{k} \lambda} \frac{\partial}{\partial \gamma_{\kappa \lambda}}+\frac{1}{4}
$$

Then Kuchar's proposal for the Hamiltonian reads Equation (6.35):

$$
\mathrm{H}_{0} \rightarrow \hat{\mathrm{H}}_{0}=-\frac{1}{2}\left(\mathrm{~L}_{\alpha \beta \mu \mathrm{v}} \frac{\partial}{\partial \gamma_{\alpha \beta} \gamma_{\mu \mathrm{v}}}+\gamma_{\kappa \lambda} \frac{\partial}{\partial \gamma_{\kappa \lambda}}+\frac{1}{4}\right)
$$

Substitution of the wave function (6.30) in the Wheeler-DeWitt equation $\hat{\mathrm{H}}_{0} \Psi=0$, with $\hat{\mathrm{H}}_{0}$ given by the previous relation, determines the constant $\mathrm{K}_{4}$. The outcome is Equation (6.36):
$\Psi=\mathrm{c}_{1} \gamma^{1 / 4}+\mathrm{c}_{2} \gamma^{-1 / 4}$

The constants $c_{1}, c_{2}$, remain arbitrary and may be fixed after the selection of a proper measure via normalizability requirements.

\subsection{Quantization of the Non Abelian Model}

In the present section, the quantization of the Non Abelian model, is exhibited. This model is a Class B since $C_{\mu v}^{\mu} \neq 0$. Class $B$ Cosmological Models, have a peculiarity; if the simplifying hypothesis of homogeneity, is inserted in the Einstein-Hilbert action, the reduced action obtained, will result in equations of motion which are, in general, not equivalent to the equations one gets by the imposition of the same hypothesis, directly on the full Einstein's Field Equations. This situation does not occur for the case of Class A models. Suppose that one adopts the canonical analysis in the framework of the Hamiltonian description. Then, the problem of the existence of a "valid" Hamiltonian (i.e., of a Hamiltonian which produces equations of motion equivalent to the corresponding Einstein equations), arises. A great many of works have dealt with the problem (Christodoulakis et al. (1996) and the references therein). The conclusion was that for Class B spacetimes, with a general scale factor matrix $\gamma_{\alpha \beta}(\mathrm{t})$, a valid Hamiltonian is not known-a serious drawback since one major aim of the Hamiltonian approach, is the quantization of the system under discussion.

Though it is extremely difficult to attack this problem, partial solutions have been given in (Christodoulakis et al., 1996). Indeed, in that work, a Hamiltonian constructed out of the scale factor matrix $\gamma_{\alpha \beta}(\mathrm{t})$ and the structure constants $\mathrm{C}_{\mu \mathrm{v}}^{\alpha}$, which resembles in form the Hamiltonian for the Class A models, is constructed and sufficient conditions on the various parts of that Hamiltonian are given, in order for it to be valid. The set of these conditions is large, the conditions themselves a little complicated and auxiliary quantities enter the scheme. But, in $2+1$ analysis, not all are needed; the equations of motion, are nothing but the derivatives of the linear constraints-a fact which simplifies the system of the conditions to be satisfied and the procedure of identifications.

Thus, following the entire procedure described in (Christodoulakis et al., 1996), the following results are obtained.

The valid Hamiltonian for the Non Abelian Model, is Equation (6.37):

$$
\mathrm{H}=\mathrm{N}(\mathrm{t})\left(\frac{1}{2} \Theta_{\alpha \beta \mu v} \pi^{\alpha \beta} \pi^{\mu v}+\mathrm{V}\right)+4 \mathrm{~N}^{\rho}(\mathrm{t}) \mathrm{C}_{\rho \alpha}^{\mathrm{k}} \gamma_{\mathrm{k} \beta}(\mathrm{t}) \pi_{\alpha \beta}
$$


where, Equation (6.38a to d):

$$
\begin{aligned}
& \Theta_{\alpha \beta \mu \nu}=\frac{1}{6 q}\left(\sum_{\alpha \beta} \gamma_{\mu \nu}+\sum_{\mu \nu} \gamma_{\alpha \beta}\right) \\
& -\frac{1}{6}\left(\gamma_{\alpha \mu} \gamma_{\beta v}+\gamma_{\alpha \nu} \gamma_{\beta \mu}\right)+\gamma_{\alpha \beta} \gamma_{\mu \nu} \\
& \sum_{\alpha \beta}=C_{\tau \xi}^{\tau}\left(C_{\phi \alpha}^{\omega} \gamma_{\omega \beta}+C_{\phi \beta}^{\omega} \gamma_{\omega \alpha}\right) \gamma^{\xi \phi} \\
& q=C_{\beta \mu}^{\alpha} C_{\alpha \mu}^{\beta} \gamma^{\mu \nu} \\
& V=-12 R-3 \Lambda
\end{aligned}
$$

The quantity $\mathrm{q}$ is scalar under the action of the automorphism group, corresponds to the unique curvature scalar of the spatial surfaces of simultaneity $\sum_{\mathrm{t}}\left(\mathrm{q}=\frac{\mathrm{R}}{8}\right)$ and thus exhibits the only true degree of freedom-as far as the 2-geometry is concerned.

The corresponding scalar (under the action of the automorphism group) Lagrangian is Equation (6.39):

$$
\mathrm{L}=\frac{1}{2 \mathrm{~N}(\mathrm{t})}\left(\Theta^{\alpha \beta \mu v} \mathrm{~K}_{\alpha \beta} \mathrm{K}_{\mu v}\right)-\mathrm{N}(\mathrm{t}) \mathrm{V}
$$

where $\Theta^{\alpha \beta \mu v}$ is the inverse of $\Theta_{\alpha \beta \mu v}$ Equation (6.40):

$$
\Theta^{\alpha \beta \mu v} \Theta_{\mu v \kappa \lambda}=\delta_{\kappa \lambda}^{\alpha \beta}
$$

Given by Equation (6.41a to k):

$$
\begin{aligned}
& \Theta^{\alpha \beta \mu \nu}=\Delta^{\alpha \beta \mu \nu}+c_{1}\left(S^{\alpha \beta} G^{\mu \nu}+S^{\mu \nu} G^{\alpha \beta}\right) \\
& +c_{2}\left(S^{\alpha \beta} S^{\mu \nu}\right)+c_{3}\left(G^{\alpha \beta} G^{\mu \nu}\right) \\
& \omega=\frac{1}{6 q} \\
& c_{1}=-\frac{\omega(1+\Gamma \omega)}{1+2 \Gamma \omega+\Gamma^{2} \omega^{2}-G S \omega^{2}} \\
& c_{2}=\frac{G \omega^{2}}{1+2 \Gamma \omega+\Gamma^{2} \omega^{2}-G S \omega^{2}} \\
& c_{3}=\frac{S \omega^{2}}{G s \omega^{2}-(1+\Gamma \omega)^{2}}
\end{aligned}
$$

$$
\Delta^{\alpha \beta \mu v}=-\frac{3}{2}\left(\gamma^{\alpha \mu} \gamma^{\beta v}+\gamma^{\alpha v} \gamma^{\beta \mu}\right)+\frac{9}{5} \gamma^{\alpha \beta} \gamma^{\mu v}
$$$$
S^{\alpha \beta}=\Delta^{\alpha \beta \mu v} \Sigma_{\mu v}
$$

$$
\mathrm{G}^{\alpha \beta}=\Delta^{\alpha \beta \mu v} \gamma_{\mu v}
$$

$S=\Delta^{\alpha \beta \mu v} \Sigma_{\alpha \beta} \Sigma_{\mu v}$

$$
\mathrm{G}=\Delta^{\alpha \beta \mu v} \gamma_{\alpha \beta} \gamma_{\mu v}
$$

$\Gamma=\Delta^{\alpha \beta \mu v} \gamma_{\alpha \beta} \Sigma_{\mu v}$

And Equation (6.42):

$\mathrm{K}_{\alpha \beta}(\mathrm{t})=\dot{\gamma}_{\alpha \beta}(\mathrm{t})-2 \mathrm{~N}^{\phi}(\mathrm{t})\left(\mathrm{C}_{\phi \alpha}^{\rho}+\mathrm{C}_{\phi \beta}^{\rho} \gamma_{\rho \alpha}\right)$

While Equation (6.43):

$\pi^{\alpha \beta}=\Theta^{\alpha \beta \mu v} K_{\mu v}$

By inversion of the equation Equation (6.44):

$\dot{\gamma}_{\alpha \beta}=\frac{\partial \mathrm{H}}{\partial \pi^{\alpha \beta}}$

Again upon quantization, following Kuchar's proposal, in the Schrodinger representation Equation (6.45):

$\frac{1}{2} \Theta_{\alpha \beta \mu v} \pi^{\alpha \beta} \pi^{\mu \nu} \rightarrow-\frac{1}{2} \square_{c}^{2}$

Where Equation (6.46):

$\square_{c}^{2}=\square^{2}=\frac{1}{\sqrt{g_{11}}} \partial_{\gamma}\left\{\sqrt{g_{11}} g^{11} \partial_{\gamma}\right\}$

is the 1-dimensional Laplacian based on the "physical metric" $g_{11}$ Equation (6.47):

$\mathrm{g}^{11}=\Theta_{\alpha \beta \mu \nu} \frac{\partial \mathrm{q}}{\partial \gamma_{\alpha \beta}} \frac{\partial \mathrm{q}}{\partial \gamma_{\mu \nu}}=\frac{2}{3} \mathrm{q}^{2}$

With $\mathrm{g}^{11} \mathrm{~g}_{11}=1$-similarly to the Abelian case. It has been mentioned that in 1-dimension the conformal group is totally contained in the G.C.T. group, in the sense that any conformal transformation of the metric cannot produce any 
change in the-trivial-geometry and is thus reachable by some G.C.T. Therefore, no extra term in needed in (6.46), as it can also formally be seen by taking the limit $\mathrm{n}=1, \mathrm{R}=$ 0 in the general definition Equation (6.48):

$\square_{c}^{2}=\square^{2}+\frac{(d-2)}{4(d-1)} R=\square^{2}$

Thus Equation (6.49):

$$
\mathrm{H}_{0} \rightarrow \hat{\mathrm{H}}_{0}=-\frac{1}{3} \mathrm{q} \partial_{\mathrm{q}}\left(\mathrm{q} \partial_{\mathrm{q}}\right)-3(4 \mathrm{q}+\Lambda)
$$

So, the Wheeler-DeWitt equation now, reads Equation (6.50):

$$
\hat{\mathrm{H}}_{0} \Psi=\frac{\mathrm{q}^{2}}{3} \Psi "(\mathrm{q})-\frac{\mathrm{q}}{3} \Psi^{\prime}(\mathrm{q})-12 \mathrm{q} \Psi(\mathrm{q})-3 \Lambda \Psi(\mathrm{q})=0
$$

The general solution to this equation, for $\Lambda \neq 0$, is Equation (6.51):

$$
\Psi(\gamma)=\mathrm{c}_{1} \mathrm{~J}_{6 \mathrm{i} \sqrt{\Lambda}}(12 \sqrt{\mathrm{q}})+\mathrm{c}_{2} \mathrm{~J}-{ }_{6 \mathrm{i} \sqrt{\Lambda}}(12 \sqrt{\mathrm{q}})
$$

where, $\mathrm{J}_{\mathrm{n}}$, is the Bessel Function of the first kind and $\mathrm{c}_{1}$, $\mathrm{c}_{2}$, arbitrary constants.

If $\Lambda$ vanishes, the solution is Equation (6.52):

$$
\Psi(\gamma)=\mathrm{c}_{3} \mathrm{~J}_{0}(12 \sqrt{\mathrm{q}})+\mathrm{c}_{4} \mathrm{Y}_{0}(12 \sqrt{\mathrm{q}})
$$

where, $Y_{n}$ is the Bessel Function of the second kind and $\mathrm{c}_{3}, \mathrm{c}_{4}$, arbitrary constants.

\subsection{The Equivalence of the State Spaces}

In our examples the classical Geometries are either Minkowski spaces or Spaces of Constant curvature, which in three dimensions implies maximal symmetry. Thus, the 3 curvature invariants suffice to characterize the space. We therefore take the following set of invariant relations Equation (6.53a to c):

$$
\begin{aligned}
& \mathrm{Q}_{1}=\mathrm{R}_{\mathrm{A}}^{\mathrm{A}} \\
& \mathrm{Q}_{2}=\mathrm{R}_{\mathrm{B}}^{\mathrm{A}} \mathrm{R}_{\mathrm{A}}^{\mathrm{B}}-\frac{\mathrm{R}^{2}}{3} \\
& \mathrm{Q}_{3}=\mathrm{R}_{\mathrm{B}}^{\mathrm{A}} \mathrm{R}_{\Gamma}^{\mathrm{B}} \mathrm{R}_{\mathrm{A}}^{\Gamma}-\frac{\mathrm{R}^{3}}{9}
\end{aligned}
$$

Now, in Gauss normal coordinates, a three dimensional spatially homogeneous metric takes the form Equation (6.54):

$\mathrm{g}_{\mathrm{AB}}=\left(\begin{array}{ccc}-1 & 0 & 0 \\ 0 & \mathrm{~g}_{11} & \mathrm{~g}_{12} \\ 0 & \mathrm{~g}_{12} & \mathrm{~g}_{22}\end{array}\right)$

where, the spatial part $\mathrm{g}_{\mathrm{ij}}$ is Equation (6.55):

$$
\mathrm{g}_{\mathrm{ij}}=\sigma_{\mathrm{i}}^{\alpha} \sigma_{\mathrm{j}}^{\beta} \gamma_{\alpha \beta}
$$

For the scale factor matrix $\gamma_{\alpha \beta}$ we assume three arbitrary functions of time, i.e., we depart from the classical solutions yet keeping ourselves within the class of spatially homogeneous three-geometries. If we now use the one-forms appropriate for the Abelian and non-Abelian symmetry group, we get, as expected from the theorem of section 2, the following results concerning the pair designated by the vanishing of the cosmological constant(containing the two cosmological parameterizations of 3d Minkowski space).

Abelian, $\Lambda=0$ Equation (6.56a to $\mathrm{c})$ :

$Q_{1}=-2 G_{0}^{0}$

$\mathrm{Q}_{2}=\frac{2}{3}\left(\mathrm{G}_{0}^{0}\right)^{2}$

$\mathrm{Q}_{3}=-\frac{10}{9}\left(\mathrm{G}_{0}^{0}\right)^{3}$

Non Abelian, $\Lambda=0$ Equation (6.57a to c):

$\mathrm{Q}_{1}=-2 \mathrm{G}_{0}^{0}$

$\mathrm{Q}_{2}=\frac{2}{3}\left(\mathrm{G}_{0}^{0}\right)^{2}-2 \gamma^{\alpha \beta} \mathrm{G}_{\alpha}^{0} \mathrm{G}_{\beta}^{0}$

$\mathrm{Q}_{3}=-\frac{10}{9}\left(\mathrm{G}_{0}^{0}\right)^{3}+3 \gamma^{\alpha \beta} \mathrm{G}_{\alpha}^{0} \mathrm{G}_{\beta}^{0}$

While, when a cosmological constant exists, the corresponding pair (containing the two cosmological parameterizations of $3 \mathrm{~d}$ Maximally Symmetric space) gives the following set of relations.

Abelian, $\Lambda \neq 0$ Equation (6.58a to c):

$\mathrm{Q}_{1}=-2\left(\mathrm{G}_{0}^{0}+\Lambda\right)$ 


$$
\mathrm{Q}_{2}=\frac{2}{3}\left(\mathrm{G}_{0}^{0}+\Lambda\right)^{2}
$$

$$
\mathrm{Q}_{3}=-\frac{10}{9}\left(\mathrm{G}_{0}^{0}+\Lambda\right)^{3}+4 \Lambda\left(\mathrm{G}_{0}^{0}+\Lambda\right)^{2}
$$

Non Abelian, $\Lambda \neq 0$ Equation (6.59a to c):

$$
\begin{aligned}
& \mathrm{Q}_{1}=-2\left(\mathrm{G}_{0}^{0}+\Lambda\right) \\
& \mathrm{Q}_{2}=\frac{2}{3}\left(\mathrm{G}_{0}^{0}+\Lambda\right)^{2}-2 \gamma^{\alpha \beta} \mathrm{G}_{\alpha}^{0} \mathrm{G}_{\beta}^{0} \\
& \mathrm{Q}_{3}=-\frac{10}{9}\left(\mathrm{G}_{0}^{0}+\Lambda\right)^{3}+4 \Lambda\left(\mathrm{G}_{0}^{0}+\Lambda\right)^{2} \\
& +3\left(\mathrm{G}_{0}^{0}+\Lambda\right) \gamma^{\alpha \beta} \mathrm{G}_{\alpha}^{0} \mathrm{G}_{\beta}^{0}-12 \Lambda \gamma^{\alpha \beta} \mathrm{G}_{\alpha}^{0} \mathrm{G}_{\beta}^{0}
\end{aligned}
$$

Now, it is a fact that $\mathrm{G}_{0}^{0}$ and $\mathrm{G}_{\alpha}^{0}$ become, upon transition to a Hamiltonian formulation, linear combinations of $\mathrm{H}_{0}$ and $\mathrm{H}_{\alpha}$. This, in turn, permits us to conclude that, upon canonically quantizing the pairs in the two cases above mentioned, there will always exist an entire host of factor orderings for each of the quantum analogues of $\mathrm{Q}_{1}, \mathrm{Q}_{2}, \mathrm{Q}_{3}$ such that their eigenvalues become 0 or $6 \Lambda$ (for $Q_{1}$ ). This establishes the equivalence of the corresponding quantum states under non-trivial space-time coordinate transformations. Note that, when an arbitrary gauge is used, the previous relations simply become more complicated; the qualitative result that $\mathrm{Q}_{1}, \mathrm{Q}_{2}, \mathrm{Q}_{3}$ are functions of the constraints, remains of course valid.

\section{DISCUSSION}

We have discussed the issue of space-time covariance of Canonical Quantization of General Relativity. To many this is known as the problem of time. At the classical level, the well established canonical analysis of the Einstein-Hilbert action leaves no room for doubts: The formulation is explicitly space-time generally covariant. Upon canonically quantizing the problem seems to reappear as all the ingredients of the theory, i.e., the quantum constraints and consequently the quantum states concern the three-geometry and not the space-time in which it is embedded. An answer to the problem presupposes a commitment about what will constitute the set of observables. Motivated by the very essence of the notion of a geometry, we adopt the point of view that this set must be identified by all invariant relations between the various curvature or higher derivative curvature scalars of a given geometry. Each such relation must be turned into an entity living on the phase space by eliminating the time derivatives of the extrinsic curvature (through use of the spatial equations of motion, if necessary). The appeal to a well known theorem of Constrained Dynamics permits us to conclude that all these entities are homogeneous polynomials of the Quadratic and the Linear constraints. Therefore, when we wish to turn each and every such entity into operator (Quantum Observable), there will be many factor orderings (namely all these that keep at least one constraint to the far right) which will enable this operator to annihilate the states defined as the common null eigenstates of the Quantum Constraints. Consequently, the use of many different slices as bases for canonically quantizing one and the same space-time can have no effect on the sol defined quantum observables. Spacetime covariance is thus observed at the quantum level. The above considerations are not meant to imply that we claim we have constructed a Dirac Consistent quantum theory of General Relativity. They rather point to the claim that, if a consistent imposition of the quantum constraints is achieved, we expect to encounter no additional problems concerning space-time covariance of the ensuing theory. In that sense, our result for full pure gravity is formal. It is seen to be explicitly realized in the case of $2+1$ cosmology considered.

\section{ACKNOWLEDGEMENT}

The authors acknowledge a vivid discussion with Prof. Rafael Sorkin, whose criticism helped them shape the notion of Quantum Equivalence. They also thank for discussion Dr. E. Korfiatis and Prof. J. Zanelli. This work has been supported by EPEAEK II in the framework of "PYTHAGORAS II- SUPPORT OF RESEARCH GROUPS IN UNIVERSITIES”.

\section{Appendix}

In this appendix, we give some useful formulae, concerning the mini-superspace. Using the results of canonical analysis in a $(n+1)$-dimensional manifold, endowed with the line element, one arrives at the notion of mini-superspace spanned by $\gamma_{\alpha \beta}$ 's (coordinates) and having as "covariant" metric the following Equation (A1):

$$
\mathrm{L}^{\alpha \beta \mu v}=\frac{1}{4}\left(\gamma^{\alpha v} \gamma^{\beta \mu}+\gamma^{\alpha \nu} \gamma^{\beta \mu}-2 \gamma^{\alpha \beta} \gamma^{\mu v}\right)
$$


While the "contravariant metric", is defined as Equation (A2):

$$
\mathrm{L}_{\alpha \beta \mu v}=\left(\gamma_{\alpha \mu} \gamma_{\beta v}+\gamma_{\alpha v} \gamma_{\beta \mu}-\frac{2}{\mathrm{n}-1} \gamma_{\alpha \beta} \gamma_{\mu \nu}\right)
$$

In the sense that Equation (A3):

$$
\mathrm{L}^{\alpha \beta \kappa \lambda} \mathrm{L}_{\kappa \lambda \mu v}=\delta_{\mu v}^{\alpha \beta} \equiv \frac{1}{2}\left(\delta_{\mu}^{\alpha} \delta_{v}^{\beta}+\delta_{v}^{\alpha} \delta_{\mu}^{\beta}\right)
$$

The "Christoffel" symbols are defined as Equation (A4):

$$
\Gamma_{k \lambda}^{\alpha \beta \mu v}=\frac{1}{2} L_{\kappa \lambda \rho \sigma}\left\{L^{\rho \sigma \mu v, \alpha \beta}+L^{\alpha \beta \rho \sigma, \mu v}-L^{\alpha \beta \mu v, \rho \sigma}\right\}
$$

where, Equation (A5):

$$
\mathrm{L}^{\alpha \beta \mu v, \rho \sigma} \equiv \frac{\partial \mathrm{L}^{\alpha \beta \mu v}}{\partial \gamma_{\rho \sigma}}
$$

Combined usage of (A.1), (A.2), (A.3) and (A.4), gives Equation (A6):

$$
\Gamma_{\kappa \lambda}^{\alpha \beta \mu v}=-\frac{1}{4}\left(\gamma^{\alpha \mu} \delta_{\kappa \lambda}^{\beta \mu}+\gamma^{\beta v} \delta_{\kappa \lambda}^{\alpha \mu}+\gamma^{\beta \mu} \delta_{\kappa \lambda}^{\alpha v}+\gamma^{\beta v} \delta_{\kappa \lambda}^{\alpha \mu}\right)
$$

In the same spirit, "Riemann" tensor is defined as follows Equation (A7):

$$
\mathrm{R}_{\kappa \lambda}^{\alpha \beta \rho \sigma \mu v}=\Gamma_{\kappa \lambda}^{\alpha \beta \rho \sigma, \mu v}-\Gamma_{\kappa \lambda}^{\alpha \beta \mu v, \rho \sigma}+\Gamma_{\omega \varepsilon}^{\alpha \beta \rho \sigma} \Gamma_{\kappa \lambda}^{\omega \xi \mu \nu}-\Gamma_{\omega \xi}^{\alpha \beta \mu v} \Gamma_{\kappa \lambda}^{\omega \xi \rho \rho \sigma}
$$

where, Equation (A8):

$$
\Gamma_{\kappa \lambda}^{\alpha \beta \mu v, \rho \sigma} \equiv \frac{\partial \Gamma_{\kappa \lambda}^{\alpha \beta \mu v}}{\partial \Upsilon_{\rho \sigma}}
$$

Contraction of $(\rho, \sigma)$ with $(\kappa, \lambda)$ results in the "Ricci" tensor Equation (A9):

$$
\mathrm{R}^{\alpha \beta \mu \nu}=\Gamma_{\kappa \lambda}^{\alpha \beta \kappa \lambda, \mu v}-\Gamma_{\kappa \lambda}^{\alpha \beta \mu \nu, \kappa \lambda}+\Gamma_{\omega \xi}^{\alpha \beta \kappa \lambda} \Gamma_{\kappa \lambda}^{\omega \xi \mu \nu}-\Gamma_{\omega \xi}^{\alpha \beta \mu v} \Gamma_{\kappa \lambda}^{\omega \xi \kappa \lambda}
$$

A lengthy but straightforward calculation, gives Equation (A9):

$$
\mathrm{R}^{\alpha \beta \mu v}=\frac{1}{8}\left(\mathrm{n} \gamma^{\alpha \mu} \gamma^{\beta v}+\mathrm{n} \gamma^{\alpha v} \gamma^{\beta \mu}-2 \gamma^{\alpha \beta} \gamma^{\mu v}\right)
$$

With the help of (A.10) and (A.2) the $\backslash$ Ricci" scalar is found to be Equation (A11):

$$
\mathrm{R}=\mathrm{L}_{\alpha \beta \mu \nu} \mathrm{R}^{\alpha \beta \mu \nu}=\frac{1}{4}\left(\mathrm{n}^{3}+\mathrm{n}^{2}-2 \mathrm{n}\right)
$$

Finally, the Iconformal Beltrami" operator, is Equation (A12):

$$
\begin{aligned}
& \square_{\mathrm{C}}^{2} \equiv \square^{2}+\frac{\mathrm{D}-2}{4(\mathrm{D}-1)}=\mathrm{L}_{\alpha \beta \mu \nu} \frac{\partial^{2}}{\partial \gamma_{\alpha \beta} \gamma_{\mu \nu}} \\
& -\mathrm{L}_{\alpha \beta \mu \nu} \Gamma_{\kappa \lambda}^{\alpha \beta \mu \nu} \frac{\partial}{\partial \gamma_{\kappa \lambda}}+\frac{\mathrm{D}-2}{4(\mathrm{D}-1)} \mathrm{R}
\end{aligned}
$$

where, $\mathrm{D}$ is the dimension of the general metric space: $\mathrm{D}=\frac{\mathrm{n}(\mathrm{n}+1)}{2}$, i.e., the number of the independent $\gamma_{\mu v}{ }^{\prime}$ s.

One can find that Equation (A13):

$\mathrm{L}_{\alpha \beta \mu v} \Gamma_{\kappa \lambda}^{\alpha \beta \mu \nu}=\frac{3-\mathrm{n}^{2}}{\mathrm{n}-1} \gamma_{\kappa \lambda}$

Thus (A.12), takes the form Equation (A14):

$$
\square_{C}^{2} \equiv L_{\alpha \beta \mu \nu} \frac{\partial^{2}}{\partial \gamma_{\alpha \beta} \gamma_{\mu \nu}}-\frac{3-n^{2}}{n-1} \gamma_{\kappa \lambda} \frac{\partial}{\partial \gamma_{\kappa \lambda}}+\frac{D-2}{4(D-1)} R
$$

\section{REFERENCES}

Christodoulakis, T., E. Korfiatis and A. Paschos, 1996. New method for constructing valid Hamiltonians for class-B spacetimes and the quantization of the general type-V cosmology. Phys. Rev. D, 54: 26912698. DOI: 10.1103/PhysRevD.54.2691

Christodoulakis, T., E. Korfiatis and G.O. Papadopoulos, 2002a. Automorphism inducing diffeomorphisms, ginvariant characterization of homogeneous 3spaces and hamiltonian dynamics of Bianchi cosmologies. Commun. Math. Phys., 226: 377-391. DOI: $10.1007 / \mathrm{s} 002200200611$

Christodoulakis, T., T. Gakis and G.O. Papadopoulos, $2002 \mathrm{~b}$. Conditional symmetries and the quantization of Bianchi Type I vacuum cosmologies with and without cosmological constant. Class. Quantum Grav., 19: 1013-1025. DOI: 10.1088/0264$9381 / 19 / 6 / 301$ 
Christodoulakis, T., G. Kofinas, E. Korfiatis, G.O. Papadopoulos and A. Paschos, 2001. Timedependent automorphism inducing diffeomorphisms in vacuum Bianchi cosmologies and the complete closed form solutions for types II and V. J. Math. Phys., 42: 3580-3580. DOI: 10.1063/1.1386637

Dirac, P.A.M., 1950. Generalized hamiltonian dynamics. Canad. J. Math., 2: 129-148. DOI: 10.4153/CJM1950-012-1

Eisenhart, L.P., 1933. Continuous Groups of Transformations. 1st Edn., Princeton Univ. Press.

Hájíček, P. and K.V. Kuchař, 1990. Constraint quantization of parametrized relativistic gauge systems in curved spacetimes. Phys. Rev. D, 41: 1091-1104. DOI: 10.1103/PhysRevD.41.1091
Isham, C., 1995. Structural issues in quantum gravity. Imperial College of Science. http://arxiv.org/pdf/grqc/9510063v1.pdf

Kuchar, K.V., 1982. J. Math. Phys., 23: 1647-1661

Milne, 1932. Nature, 130: 9.

Sneddon, G.E., 1976. Hamiltonian cosmology: A further investigation. J. Phys. A, 9: 229-229. DOI: 10.1088/0305-4470/9/2/007

Sundermeyer, K., 1982. Constrained Dynamics. 1st Edn., Springer-Verlag, Berlin, ISBN-10: 0387119477, pp: 318. 